\title{
A busca e o eye tracking: um olhar semiótico sobre o knowledge graph
}

\author{
Silvana Drumond Monteiro \\ Doutora; Universidade Estadual de Londrina, Londrina, PR, Brasil; \\ silvanadrumond@gmail.com \\ Cecílio Merlotti Rodas \\ Doutor; Instituto Federal de São Paulo, São Paulo, SP, Brasil; \\ cecilio.rodas@gmail.com \\ Silvana Aparecida Borsetti Gregório Vidotti \\ Doutora; Universidade Estadual Paulista, Marília, SP, Brasil; \\ svidotti@gmail.com
}

\begin{abstract}
Resumo: O eye tracking é, ao mesmo tempo, uma tecnologia e método de pesquisa utilizado em várias áreas do conhecimento e, recentemente, pela Ciência da Informação. Sua tese principal, formulada por Just e Carpenter (1976), é o strong eye-mind, ou seja, a forte relação entre visualização e processamento da informação. Nessa direção, foi feita uma pesquisa básica, com delineamento experimental, visando avaliar o comportamento de busca em uma Search Engine Results Page do Google, em especial a visualização do Knowledge Graph - ou mapa do conhecimento. A premissa da investigação pautou-se na semiótica peirceana, na qual os signos em um resultado de busca em maior ou menor medida - podem influenciar escolhas, qualificar resultados, capturar o olhar e ser importantes ou determinantes em uma escolha a partir de uma busca, explicando assim o strong eye-mind. Os resultados demonstraram que a imagem é um atrator cognitivo; no entanto, a escolha depende também de outros fatores, como o tipo de busca e o hábito do sujeito em pesquisas nos mecanismos de busca.
\end{abstract}

Palavras-chave: Eye Tracking. Semiótica peirceana. Knowledge Graph. Google. Taxonomia da busca

\section{Introdução}

O rastreamento do olhar (eye tracking), conhecido e utilizado em várias áreas, como a Psicologia, Medicina, Linguística Cognitiva, Marketing, Comunicação, Design, Computação, Engenharia e Fonoaudiologia, tem sido recentemente abordado na Ciência da Informação (CI) pelos autores Rodas, Marcos, Vidotti (2014), Rodas e Vidotti (2015, 2016), Rodas, Vidotti e Monteiro (2016a, 2016b), Rodas et al. (2016), Vidotti et al. (2016). 
Seu uso, apesar de aplicações diferenciadas para as áreas supracitadas (com proeminência nos setores comerciais), tem como objetivo estudar o processamento da informação para as mais variadas análises, como a avaliação de produtos, usabilidade da informação, comportamento de leitura, websites, entre outras.

Seu emprego na CI vem ao encontro dos estudos que fazem interfaces, por exemplo, com a Arquitetura da Informação, Experiência do Usuário - User Experience (UX), Usabilidade, Encontrabilidade, interfaces de busca (mecanismos e sistemas de recuperação da informação), avaliação de websites de bibliotecas, arquivos, museus e outros sistemas de informação e comportamento de busca. Pesquisas de Marcos e González-Caro (2010), OrtizChaves et al. (2014) e Maynes e Everdell (2014) com o eye tracking mostraram que a interface dos mecanismos pode influenciar o comportamento dos sujeitos na busca de informações, o que motivou a aplicação dessa tecnologia nos testes aqui apresentados.

A hipótese do eye tracking, formulada por Just e Carpenter (1976), ambos americanos, é o strong eye-mind, ou seja, "De acordo com esta teoria 'não há nenhum atraso entre o que é fixado e processado'. Isto é, a visualização de uma palavra ou objecto tende a ser acompanhada por um processo cognitivo.” (BARRETO, 2012, p. 170-1).

É importante observar que o eye tracking cumpre um importante papel como método de pesquisa, uma vez que fornece diversas métricas para análises e intepretações, mas deve ser acompanhado por uma teoria que seja capaz de explicar os fenômenos observados nas visualizações realizadas (coletas). Nessa direção, há repercussão com a semiótica peirciana por ela ser uma teoria sobre o poder de "representação" dos signos em uma mente (não necessariamente biológica).

Sendo assim, neste estudo, a tecnologia do eye tracking foi aplicada em uma amostra, visando a avaliar a interface de busca do Google, em especial a visualização do Knowledge Graph (KG), ou mapa do conhecimento, elemento híbrido tanto em linguagem quanto em fontes de informação, localizado, 
principalmente no canto superior direito de uma Search Engine Results Page (SERP), em uma tarefa de busca.

Para análise das informações, buscou-se esboçar, a partir da semiótica peirceana, explicações sobre o processamento das informações, especialmente as híbridas e visuais, possíveis e visualizáveis no $\mathrm{KG}$, para analisar e inferir alguns resultados sobre as escolhas dos usuários em uma busca. Nota-se que outros desdobramentos da pesquisa foram trabalhados em outros textos e, dada a extensão dos resultados, optou-se neste artigo por um olhar semiótico, mesmo que breve, como possibilidade de análise em pesquisas que utilizem o eye tracking (RODAS; VIDOTTI; MONTEIRO, 2016a, 2016b). Justifica-se a abordagem semiótica por entender que a teoria do signo pode explicar alguns fenômenos sobre escolhas, a partir da observação do movimento dos olhos, de usuários de interfaces digitais. Assim, o corpus metodológico escolhido foi o Knowledge Graph, implantado pelo Google, em língua portuguesa, no começo de 2013

O KG, como mencionado, é uma importante ferramenta que utiliza padrões semânticos advindos da Wikidata e da Wikipédia, exibindo, para o leitor, imagens e informações enciclopédias (imagens e verbetes), e tem como objetivo "prender" o leitor na página do Google, sem precisar acessar links ou consultar muitas entradas da SERP.

A semiótica peirceana, além de constituir-se em uma Ciência Geral do Signo, é capaz, por meio de seu substrato lógico, de possibilitar a interpretação desses fenômenos sígnicos e do processamento ou ação do pensamento-signo na mente (no caso, humana) ${ }^{1}$, pois:

A semiótica peirceana é, antes de tudo, uma teoria sígnica do conhecimento, que desenha, em um diagrama lógico, [...] para se pensar as eternas e imemoriais interrogações acerca da realidade e da verdade. (SANTAELLA, 2004, p. 90).

Nesse contexto, o recorte será especialmente a classificação dos signos e da imagem, respectivamente (PEIRCE, 1931, 1902, 2010; SANTAELLA, 1983, 2004, 2005; SANTAELLA; NÖTH, 2009). Dito isso, explicita-se que o artigo não se trata do estudo de comportamento de usuário (no sentido de aporte teórico tradicional da área de CI), mas sim de uma investigação sobre a busca de 
informação na interface do Google, em especial no KG com escopo na Semiótica, como citado. Em linha teórica, foi empregada também a taxonomia de busca de Broder (2002) no experimento, que categoriza as intenções da busca em: informacional, navegacional e transacional. Assim, a análise do funcionamento de sistemas semióticos poderá revelar outras perspectivas para a CI sobre o entendimento dos mecanismos de busca e as intenções de busca.

\section{Explicando o eye tracking}

$\mathrm{O}$ rastreamento do olhar teve sua origem antes das atuais tecnologias de medição. Em um breve retrospecto histórico, Barreto (2012) afirma que essa técnica iniciou com a observação direta do movimento ocular pelo oftalmologista francês Louis Émile Javal, no final do século XIX. Seu objetivo era analisar e descrever o movimento dos olhos durante o ato da leitura; ele descobriu que os olhos se movimentam por saltos (sacadas ou movimentos sacádicos) e fixações, ao contrário de varredura linear, como se imaginava à época. Foi em 1901 que a primeira técnica de eye tracking, com aplicação de luz refletida na córnea, foi aplicada por Dodge e Cline (BARRETO, 2012).

A partir disso, dois estudos foram importantes para o desenvolvimento do método: o primeiro foi a construção de um modelo matemático para o movimento humano (do olhar) por Fitts, em 1954; e, em 1960, Mason formalizou o método de análise através do centro da pupila e da reflexão da córnea para observar a fixação do olhar. "Em outras palavras, a posição ocular, a reflexão da córnea e o centro da pupila são usados como factores de rastreio ocular." (BARRETO, 2012, p. 172).

A partir da hipótese strong eye-mind de Just e Carpenter, de 1976, que associa o movimento ocular com os processos cognitivos, o desenvolvimento tecnológico dos equipamentos, softwares e algoritmos de medição eye tracking apresenta-se como importante método de pesquisa para determinadas áreas ou serviços.

Em 1988, de acordo com Barreto (2012), foi desenvolvido o primeiro sistema de eye tracking baseado em computador (PC), juntamente com 
algoritmos avançados de processamento de imagem, pela equipe de Dixon Cleveland (fundador da LC Technologies).

O principal dispositivo em um sistema de eye tracking é conhecido como eye tracker e consiste em:

[...] um dispositivo que emite raios infravermelhos para os olhos de usuário - a luz infravermelha é usada para evitar o desconforto do usuário com uma luz forte - que por sua vez batem na pupila e voltam ao dispositivo, permitindo calcular com precisão para onde está a olhar e ainda medir os movimentos oculares como as fixações, sacadas e regressões. (BARRETO, 2012, p. 172).

Atualmente, existem dois tipos de dispositivos, os vestíveis (wearable) e os remotos. Os dispositivos vestíveis possuem o formato de óculos, possibilitando que o sujeito se locomova livremente enquanto visualiza os objetos de interesse. Isso permite, por exemplo, o registro dos olhares sobre produtos dispostos em gôndolas de supermercados (ambiente dinâmico). Os dispositivos remotos permanecem fixos entre o sujeito e o objeto de estudo (estímulo) e são normalmente utilizados para registrar o seu olhar em interfaces exibidas em telas de computadores, sendo que alguns modelos já permitem o registro em telas de smartphones e/ou tablets. Antes do início de cada uma das seções de testes, é necessário que seja feita a calibração, pois se trata de um procedimento que permite o ajuste do equipamento para que possa rastrear e gravar o olhar do sujeito sobre a tela na qual serão exibidos os estímulos.

De acordo com Holmqvist et al. (2011), a tecnologia de Eye Tracking pode fornecer mais de 100 métricas; no entanto, as infracitadas são as mais utilizadas a partir da definição de uma Área de Interesse (AOI - Area of Interest), com o uso do equipamento e o software Tobii Studio (utilizado nesta pesquisa) (ARRAZOLA; MARCOS, 2014; POOLE; BALL, 2006; TOBII, 2015):

a) Time to First Fixation (TFF) ou tempo para a primeira fixação (segundos): métrica capaz de identificar o tempo que um sujeito leva para fixar seu olhar pela primeira vez em uma determinada AOI. Segundo Poole e Ball (2006), se uma AOI é visualizada rapidamente, isso significa que ela possui boa qualidade quanto às propriedades referentes à atenção;

b) Fixations Before (FB) ou fixações anteriores (unidade): número de fixações que foram realizadas pelos sujeitos antes de chegar a uma determinada AOI. Semelhante ao item anterior, quanto menos fixações 
forem feitas até que se visualize a AOI objeto de estudo, melhor será a qualidade quanto às propriedades referentes à atenção;

c) First Fixation Duration (FFD) ou duração da primeira fixação (segundos): expressa o tempo de duração da primeira fixação em uma AOI;

d) Fixation Duration (FD) ou duração da fixação (segundos): duração de cada uma das fixações em uma AOI objeto de estudo. Um tempo muito grande de fixação pode significar dificuldade para compreender a informação contida em uma determinada área (AOI), ou então interesse;

e) Total Fixation Duration (TFD) ou duração total da fixação (segundos): mostra o tempo total que os sujeitos de pesquisa se dedicaram olhando uma determinada AOI. Dependendo da tarefa, pode revelar valores referentes à dificuldade de compreensão ou interesse;

f) Fixation Count (FC) ou contagem das fixações (unidade): mostra a quantidade de fixações sobre uma AOI. Dependendo da tarefa, se o número de fixações global for muito grande, isso pode significar dificuldades para encontrar informação. Se uma ou mais áreas do estímulo possuírem maior número de fixações, isso pode também revelar o nível de interesse dessas áreas;

g) Visit Duration (VD) ou duração da visita (segundos): expressa o tempo da duração dos olhares em uma determinada AOI a cada vez que o sujeito a visualiza. Semelhante ao tempo de fixação, dependendo da tarefa dada, essa métrica está relacionada ao interesse ou dificuldade para a compreensão do conteúdo;

h) Total Visit Duration (TVD) ou duração total da visita (segundos): identifica o tempo total que um sujeito permaneceu olhando uma AOI durante todo o período de uma determinada sessão do teste;

i) Visit Count (VC) ou contagem de visitas (unidade): número de vezes que o olhar se dirigiu para uma AOI. Dependendo da tarefa, esse valor pode significar interesse ou problemas de visibilidade;

j) Percentage fixated (\% Fix) ou porcentagem fixada (porcentagem): porcentagem de pessoas que olharam para uma determinada AOI. Essa métrica está relacionada a questões referentes à visibilidade; assim, se uma AOI é considerada importante dentro de um determinado estímulo, e se ela é pouco visualizada, isso pode significar que a interface deveria ser modificada;

k) Percentage clicked (\% Click) ou porcentagem clicada (porcentagem): expressa a porcentagem de sujeitos que clicaram sobre uma determinada AOI. Quanto mais pessoas clicarem sobre uma AOI objeto de estudo, maior será a taxa de sucesso na execução de uma tarefa;

1) Time from First Fixation to next mouse Click (TFFC) ou tempo da primeira fixação até o primeiro clique do mouse: mostra o tempo que 
passa a partir do momento que o sujeito olha pela primeira vez para uma AOI até o momento em que ele "clica" nela;

m) Time to First mouse Click (TFC) ou tempo para o primeiro clique do mouse (segundos): indica o tempo decorrido desde o início de uma seção de teste até o momento em que uma pessoa clica sobre uma AOI. Essa métrica pode estar relacionada a questões de visibilidade referentes a uma AOI de interesse;

n) Mouse Click Count (MCC) ou contagem dos cliques do mouse (unidade): número total de cliques que uma determinada $\mathrm{AOI}$ recebeu durante uma sessão do teste.

Não menos importantes são as medidas relacionadas ao mapa do olhar (gazeplot), que ilustram as fixações e as sacadas, movimentos produzidos entre uma fixação e outra; e o mapa de calor (heatmap), que representa, por meio da cor, o conjunto de fixações realizadas e o tempo de duração em cada área da interface, sendo que as cores consideradas mais quentes, como o vermelho, laranja e amarelo, por exemplo, representam as áreas com maior incidência de olhares, e as cores consideradas mais frias, como o verde e azul, representam locais com menos visualizações (VIDOTTI et al., 2016). Segundo Rodas et al. (2016, p. 3796) “[...] tais mapas são capazes de fornecer uma primeira visão geral sobre o comportamento do olhar dos usuários sobre o estímulo.” (ver Figura 1).

Figura 1 - Exemplo de mapa de olhar (gazeplot) e mapa de calor (heatmap), respectivamente

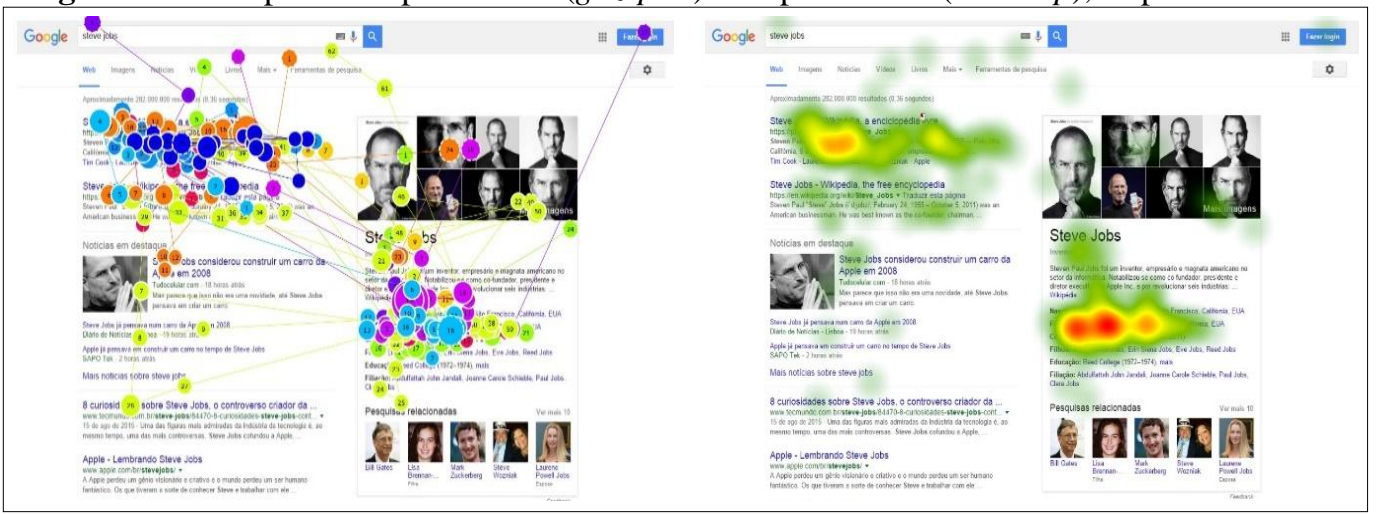

Fonte: Elaborado pelos autores

\section{O Knowledge Graph e a Semiótica}

No Brasil, na área de Ciência da Informação, são poucos os pesquisadores que estudam o Knowledge Graph (MONTEIRO, 2014, 2015; MONTEIRO; 
MOURA, 2016; SANTARÉM SEGUNDO; SOUSA; CONEGLIAN; 2015). No blog oficial do Google (2012, online), o Knowledge Graph é descrito como “[...] um enorme mapa de elementos do mundo real e as conexões entre eles, de modo a oferecer resultados mais relevantes." Em seu lançamento, três de seus atributos foram apresentados: encontrar as coisas certas; conseguir os melhores resumos; ir de forma mais ampla e profunda na busca (GOOGLE, 2012).

O KG pode ser considerado um sistema de organização, um misto de índice e conhecimento enciclopédico, que fornece, em uma busca, elementos em contexto tanto do sujeito pesquisador quanto da palavra-chave (query), a partir de consulta em várias fontes referenciais (Wikipédia) e em banco de dados estruturados ou semânticos (Wikidata ${ }^{2}$ ). É resultado de pesquisas avançadas em Recuperação da Informação e Inteligência Artificial, especialmente do Google, que tem como objetivo a aprendizagem da máquina e a semantização dos resultados de busca.

Não obstante, faltava avaliar em que medida o sujeito comum - sem o conhecimento científico dessas tecnologias e teorias - sentia, em suas decisões informacionais, o quão rico ou relevante é esse elemento na recuperação de uma informação. Dessa forma, buscou-se, na teoria Semiótica, alguns indícios que possam interpretar as escolhas, o movimento do olhar e a apreensão dos signos que constavam nos resultados de busca.

Para Monteiro et al. (2011, p. 2536),

[...] a plataforma Web é o ambiente contemporâneo para acesso à informação e ao conhecimento, dispersos no ciberespaço, e os mecanismos tecnológicos para a sua realização fazem parte desse contexto.

Hoje, a busca é um fenômeno social e Broder (2002), ao estudá-la, estabeleceu uma taxonomia, isto é, os tipos de buscas que as pessoas fazem ao utilizar os mecanismos: a busca informacional, a navegacional e a transacional.

$\mathrm{Na}$ informacional, os mecanismos são utilizados como ferramentas para consulta de uma determinada informação, a partir de uma determinada query, que no KG transformou-se em tags semânticas. Nesse sentido, esses mecanismos estão conseguindo evoluir de uma gramática de palavra-chave para 
uma linguagem com contexto semântico (designada na área de computação como "linguagem natural").

$\mathrm{Na}$ busca navegacional, o objetivo é localizar um website que já foi visitado ou localizar uma informação que o sujeito pesquisador acredita que exista na web. Na verdade, para Batelle (2006), esse tipo de query, que se utiliza do mecanismo como um browser, é chamado "pergunta piloto".

Já a busca transacional tem como função realizar alguma atividade mediada pela web, seja usar um website de serviço, realizar uma compra, entrar em um catálogo, entre outras.

Broder (2002) afirma que a evolução dos mecanismos proporcionará a apresentação de diversas fontes com o objetivo de contextualizar o indivíduo no que diz respeito à sua busca e, ainda, compreender a sua necessidade por meio da análise semântica, determinação do contexto, entre outros aspectos.

Dessa forma, o KG realiza as premissas de Broder (2002) em sua forma de apresentação. É importante salientar que o KG (e o Google) também apresentam elementos pragmáticos dos indivíduos, isto é, marca suas trilhas informacionais (índices), seus hábitos, os logs com históricos de busca e atua como um interpretante, enfim, aspectos relacionados à semiótica peirceana.

Monteiro (2015), nesse universo de investigação e a propósito do fundamento do signo, descreve esse processo de redes semântico-semióticas digitais com os seguintes elementos: objetos digitais; signos (tags semânticas e queries); e interpretantes (Knowledge Graph e sujeitos interpretantes).

Para a análise desta pesquisa, foi empregada a classificação do signo, especialmente quanto à relação do signo $\left(2^{\circ}\right)$ com seu objeto de representação, ou seja: ícone, índice e símbolo.

A tríade ícone, índice e símbolo diz respeito primariamente à distinção entre três espécies de identidades semióticas que um signo pode ter em razão de três espécies de relações em que o signo pode estar para com o objeto, como signo desse objeto. (SANTAELLA, 2004, p. 109).

A classificação do signo realizada por Peirce (1931) segue o engendramento lógico de suas categorias fenomenológicas (Primeiridade, Secundidade e Terceiridade), isto é, das tríades que foram predicadas como 
semelhança, referência e representação e posteriormente foram fixadas como qualidade, relação e mediação. "A Primeiridade é predominante na ideia de frescor, vida e liberdade." (PEIRCE, 1931, CP 1.302)³. "A ideia de Segundo é predominante nas ideias de causalidade e força estática.”. “[...] $\mathrm{Na}$ ideia de realidade, a Secundidade é predominante.” (PEIRCE, 1931, CP 1.325). "Algumas das ideias de Terceiridade são: a generalidade, infinidade, continuidade, difusão, crescimento e inteligência." (PEIRCE, 1931, CP 1.340).

Dessa forma, existem signos que agem cognitivamente apresentando qualidades de seus objetos, seja por semelhança da forma, quando o ícone está atualizado, no caso de uma imagem (que é um hipoícone de primeiridade), seja como forma de sentimento, quando o ícone é uma cosa mentale, pura virtualidade (SANTAELLA, 2004). O índice apresenta seus objetos por meio das relações de causalidade, ação e reação, de contiguidade (índices naturais) e de referência (índices artificiais). Por fim, o símbolo atua na palavra trazendo o processo cognitivo para a reflexão, inteligibilidade e interpretação.

Achamos que esses signos, em maior ou menor medida, em um resultado de busca (SERP), podem influenciar escolhas, qualificar resultados, capturar o olhar, enfim, ser importantes ou determinantes em uma escolha a partir de uma busca e assim explicar o strong eye-mind, "Isto é, [que] a visualização de uma palavra ou objecto tende a ser acompanhada por um processo cognitivo." (BARRETO, 2012, p. 170-1). Nesse sentido,

[...] o simples ato de olhar já está carregado de interpretação, visto que é sempre o resultado de uma elaboração cognitiva, fruto de uma mediação sígnica que possibilita nossa orientação no espaço por um reconhecimento e assentimento diante das coisas que só o signo permite. (SANTAELLA, 1983, p. 79-80).

Sendo assim, as próximas seções apresentam o experimento e a análise dos resultados.

\section{0 experimento}

Esta pesquisa é considerada básica, isto é, tem por finalidade gerar novos conhecimentos, de delineamento ou tipo experimental, uma vez que foi realizado um teste com a tecnologia eye tracking em alguns sujeitos de pesquisa. 
A pesquisa experimental ou de laboratório, “[...] diferentemente de uma pesquisa bibliográfica ou de campo, permite ao pesquisador manipular suas variáveis, isolá-las ou até mesmo provocar eventos passíveis de controle." (CRUZ; RIBEIRO, 2004, p. 21). Apesar do empirismo, ela não se confunde com pesquisa aplicada, embora possa ser a primeira etapa para esta última. Para Witter e Paschoal (2010, p. 135), as pesquisas experimentais estariam dentro do grupo de investigações inferenciais e são mais complexas, pois "[...] requerem mais capacitação do pesquisador, mas garantem maior controle, conhecimento e generalização e uso dentro de uma dada margem de segurança.”.

Em relação aos sujeitos de pesquisa, são 31 estudantes dos cursos de Biblioteconomia e Arquivologia da Unesp, campus de Marília, e dos cursos de Engenharia Civil e Análise de Desenvolvimento de Sistemas do Instituto Federal de São Paulo, campus Votuporanga, que se dispuseram a participar da investigação ${ }^{4}$. O primeiro teste foi aplicado em 2015 (dez estudantes), e o segundo em 2016 (21 estudantes). Quanto à amostra, Duckowisk (2007) afirma que testes dessa natureza, com muitas pessoas ou tarefas, podem demandar muito tempo e torná-los inviáveis.

A tarefa teve o intuito de verificar o comportamento de busca desses sujeitos diante de uma query do tipo informacional e navegacional (2015 e 2016, respectivamente) em um conjunto de resultados que continham, entre outros, o Knowledge Graph. Vale observar que, para subsidiar a discussão dos dados, foram criadas AOI (Areas of Interest, Figura 2) e, a partir da definição dessas áreas, fez-se uso das seguintes métricas: mapa de calor (heatmap, Figura 3); Time to First Fixation (TFF); Percentage fixated (\% Fix) e Percentage clicked (\% Click, ambas no Quadro 1).

A busca informacional foi requerida solicitando: Qual a data de morte de Steve Jobs?; explicando melhor, a questão motivava à navegação justificouse para avaliar a escolha de um resultado que permitisse ir diretamente a um website oficial ou mesmo navegar por várias fontes a fim de obter mais detalhes, uma vez que a questão solicitada não estava explicitada no resultado.

Assim, foi criada a seguinte tarefa: na próxima página de resultados de busca, imagine que você esteja procurando: conhecer mais detalhes da vida e 
carreira de Steve Jobs, fundador da Apple. Quando encontrar o resultado que possa conter a informação pesquisada, clique sobre o link apenas se achar necessário. Em seguida, você poderá fechar o navegador.

Para a análise dos dados, foram criadas três AOI (Areas of Interest), como pode ser visto na Figura 2, a saber:

(1) AOI-1 continha um resultado (link) orgânico (ou seja, não patrocinado) que apontava para o mesmo resultado a partir do qual o conteúdo do Knowledge Graph havia sido elaborado: a página da Wikipédia;

(2) A AOI-2 continha resultados orgânicos, um elemento conhecido como "notícias em destaque", um resultado com rich snippet ${ }^{5}$;

(3) A AOI-3 continha o Knowledge Graph (KG) na íntegra.

Figura 2 - Áreas de interesse que contêm o resultado com Knowledge Graph

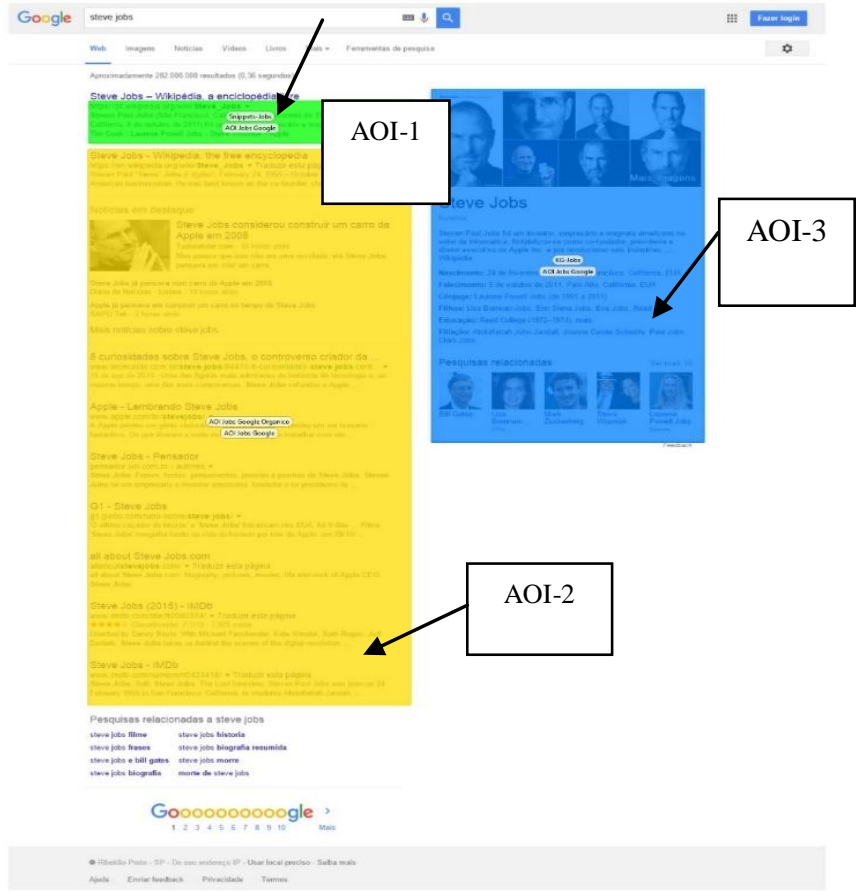

Fonte: elaborado pelos autores.

O intuito nesse teste foi verificar como esses sujeitos reagiriam diante de diferentes tarefas. Na tarefa de 2015, os estudantes foram orientados a clicar em um link somente se sentissem a necessidade de tal ação. Por se tratar de uma busca do tipo informacional, na qual se deveria procurar simplesmente pela data de falecimento de Steve Jobs, procurou-se saber se o sujeito de pesquisa seria 
capaz de confiar nas informações contidas nos snippets dos resultados ou no KG, contidos na SERP.

O KG e o primeiro resultado da SERP em questão apresentavam resultados advindos de uma mesma fonte, a Wikipédia, porém apresentados de maneira diferente. Se esse sujeito "clicasse" sobre um dos dois elementos, ele seria encaminhado para a mesma página da Wikipédia.

\section{Análise dos resultados}

A Figura 3 apresenta os mapas de calor para os dois testes realizados. No item (a), apresenta-se o mapa de calor para o teste realizado em 2016 para uma busca do tipo navegacional, na qual o sujeito de pesquisa deveria conhecer mais detalhes da vida e carreira de Steve Jobs, fundador da Apple No item (b), apresenta-se o mapa de calor para a busca do tipo informacional, procurando saber a sua data de falecimento, conforme mencionado.

Figura 3 - Mapa de calor (heatmap) referente a diferentes tarefas

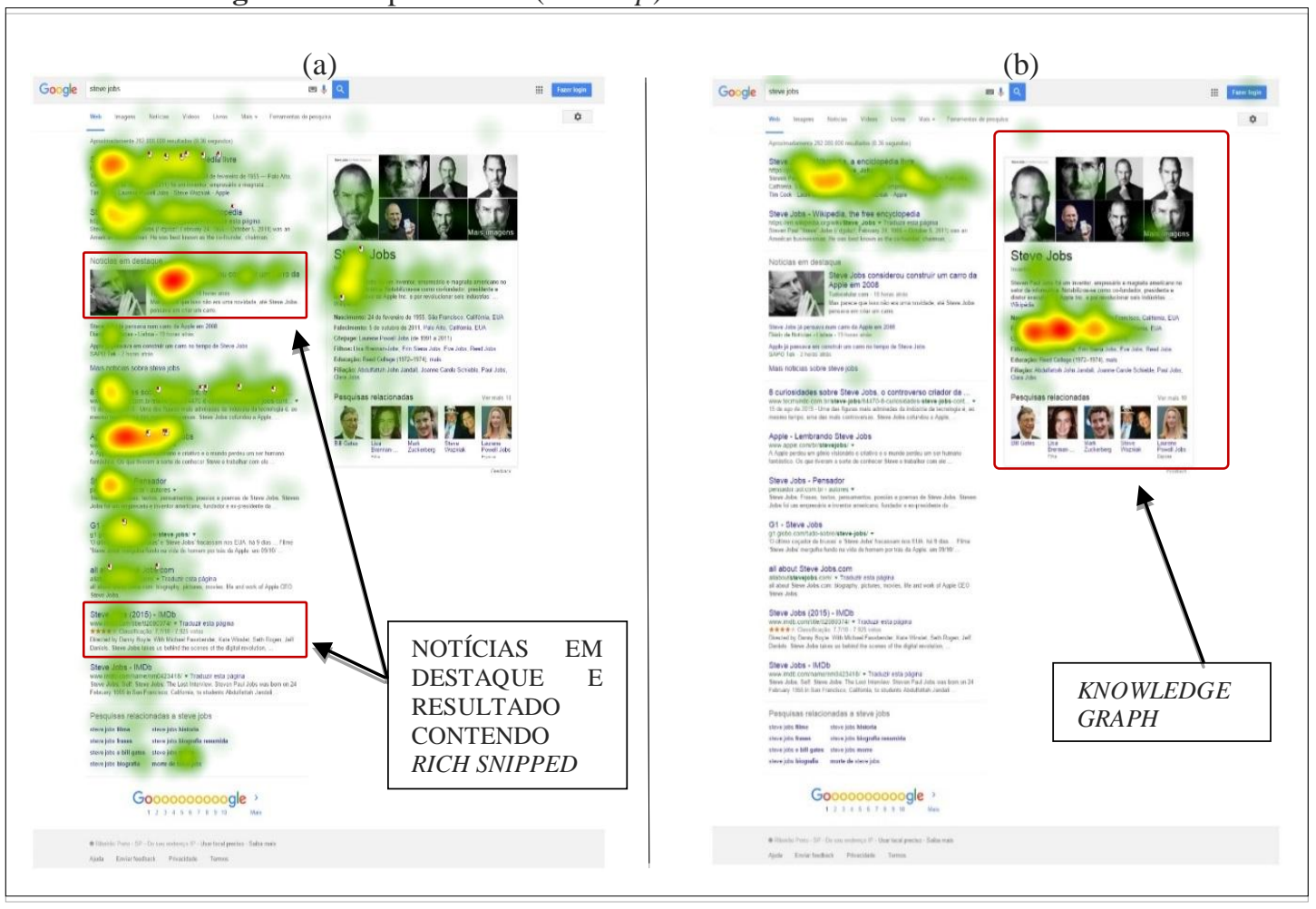

Fonte: elaborado pelos autores.

Conforme explicitado anteriormente nas métricas, o heatmap representa, por meio da cor, o conjunto de fixações realizadas e o tempo de duração em cada área da interface, sendo que as cores consideradas mais quentes, como o 
vermelho, laranja e amarelo, por exemplo, representam as áreas com maior incidência de olhares.

Nota-se que, conforme a Figura 3 (b) e o Quadro 1 (b), no teste realizado em 2015, não houve clique sobre os resultados apresentados na SERP, mostrando que os sujeitos de pesquisa confiaram nas informações contidas nos elementos, pois a data de falecimento (b) estava explícita tanto no primeiro resultado (do índice) como no KG, os dois mais visualizados, conforme Figura 3 (b).

Dessa forma, parece evidente que o tipo de busca influencia na escolha do resultado, sendo que a imagem, como atrator cognitivo, não tem muita importância no caso da busca informacional com a correspondência de uma data, uma vez que, nas zonas de calor em destaque, não há imagens, conforme (a) e (b), apesar de existirem as fotos de Steve Jobs, tanto no índice (notícias em destaque contendo rich snippet) quanto no KG. Os números são índices tão ou mais universais que as imagens, pois, para Peirce (1902, CP 2.311), "Um índice [número] pode muito bem representar a si mesmo.".

Em testes comparativos realizados com a ocorrência de rich snippets em uma busca informacional (nesse caso sem datas, mas com receita de bolo), os resultados confirmaram a proeminência das imagens (fotos e estrelas de avaliação) na escolha dos sujeitos pesquisados, de acordo com Rodas e Vidotti (2016). A imagem transita entre a referência indicial e a correspondência icônica com o seu objeto (semelhança dos hipoícones). Santaella e Nöth (2009, p. 197) indagam-se: “Qual é o potencial semiótico de uma imagem?”.

De um ponto de vista semântico, uma imagem verdadeira deve ser aquela que corresponde aos fatos que representa. De um ponto de vista sintático, deve ser aquela que representa um objeto e transmite um predicado sobre este. Do ponto de vista pragmático, deve haver uma intenção [...] por parte do emissor da mensagem pictórica.

No caso de fotos (signo mais próximo da realidade, portanto um hipoícone com proeminência indicial), Peirce (1902, CP 2.315) afirma que um “[...] índice envolve a existência de seu Objeto.”, assim as imagens trazem, em um só golpe, o objeto de busca, mesmo que esteja em um contexto híbrido. 
Pode-se inferir também que a imagem antecipa a cognição pelo seu processamento rápido e referencial.

Já no teste realizado em 2016 para uma busca navegacional, a relação dos cliques por AOI pode ser visualizada na Tabela 1 (a); na Tabela 1 (b), referente ao teste realizado em 2015 (busca informacional), verifica-se que não houve cliques. Vemos também a porcentagem dos sujeitos que visualizaram cada uma das AOI.

Tabela 1 - Dados referentes à atenção do olhar dos sujeitos

\begin{tabular}{l|c|c|c|c|c|c}
\hline & \multicolumn{3}{|c|}{$\begin{array}{c}\text { (a) 2016 } \\
\text { Conhecer mais detalhes } \\
\text { da vida e carreira de } \\
\text { Steve Jobs }\end{array}$} & \multicolumn{2}{c}{ (b) 2015} \\
& \multicolumn{3}{|c|}{$\begin{array}{c}\text { Data de falecimento de } \\
\text { Steve Jobs }\end{array}$} \\
\cline { 2 - 8 } & AOI-1 & AOI-2 & AOI-3 & AOI-1 & AOI-2 & AOI-3 \\
\hline "Cliques" & 7 & 12 & 2 & - & - & - \\
\hline Percentagem dos “cliques" (\% click) & $33,3 \%$ & $57,2 \%$ & $9,5 \%$ & - & - & - \\
\hline
\end{tabular}

Fonte: Elaborado pelos autores.

Quando comparamos a quantidade de cliques realizados sobre cada uma das AOI (a) com a quantidade total de cliques realizados durante todo o teste, observamos que a AOI-1 recebeu 33,3\% de todos os cliques, a AOI-2 recebeu 57,2\% e a AOI-3 recebeu 9,5\%. Apesar de apresentarem informações oriundas do mesmo website, ou seja, da Wikipédia, a AOI-1 recebeu maior quantidade de cliques do que a AOI-3 na busca navegacional, cerca de três vezes e meia a mais. Esse resultado referente aos dois únicos cliques sobre o $\mathrm{KG}$ parece mostrar que os sujeitos ainda parecem não se sentir seguros para escolhê-lo, ou talvez tenham dúvidas sobre onde encontrar o link dentro do elemento, que poderia direcioná-los para o website de onde se originaram as informações, ou, ainda mais, que a função enciclopédica do KG no Google está atingindo seu 
objetivo, isto é, fornecer conteúdo o suficiente para o leitor permanecer na sua página, sem a necessidade de navegação para ter acesso ao conhecimento direto na fonte.

Pela reação dos sujeitos diante do elemento $\mathrm{KG}$, ainda no levantamento de 2016, pode-se inferir que eles ou não conseguiram identificar a existência de um link que permitiria a obtenção de mais informações sobre Steve Jobs, ou tiveram dificuldades para entender as informações inseridas dentro desse elemento. Nota-se, por exemplo, que dos dois cliques realizados dentro da área referente ao KG, apenas um deles foi sobre o link, o outro foi sobre o nome do personagem, Steve Jobs, logo após as imagens no início do KG, como é possível ver na Figura 3 (a), na área referente ao KG. O nome do personagem Steve Jobs não possuía hiperlink, portanto o sujeito de pesquisa não seria capaz de ser redirecionado para o website.

Outra inferência, a partir da semiótica peirceana, pode ser feita: como o KG é um elemento relativamente novo, as pessoas, em geral, mesmo acostumadas a utilizar o Google (como no caso dos sujeitos de pesquisa), talvez não tenham o hábito de seu uso. Aqui, na acepção Semiótica, “A regra ou hábito é um padrão de ações que, sob certas condições apropriadas, será repetido indefinidamente no futuro." (SAVAN, 1976, p. 44) e pressupõe uma familiaridade, um conhecimento colateral anterior.

Ressaltando a impressão supracitada sobre o hábito, a métrica Time to First Fixation comprovou que parte dos sujeitos inicia a visualização a partir dos links tradicionais com snippets para depois visualizarem o KG; é questão de, praticamente, centésimos de segundos. Ademais, nem todos os investigados visualizaram todas as AOI, mas, quando foi possível comparar, observou-se que o início de suas pesquisas visuais na SERP começou, aparentemente, sobre os links tradicionais com rich snippets. Dessa forma, por meio da métrica TFF, que mede o tempo necessário para que o olhar do sujeito visualizasse (fixasse) pela primeira vez uma das três AOI, observou-se que apenas $10 \%$ dos sujeitos iniciaram suas visualizações/fixações pela AOI-3, ou seja, pelo KG. No segundo teste, cerca de $14 \%$ dos sujeitos iniciaram a busca pela AOI referente ao KG. 
Futuros testes poderão ser aplicados com o intuito de verificar se os sujeitos conseguiriam perceber que existe um link que os encaminha para um website, a partir do qual as informações foram geradas. Além do mais, se perceberiam todo o contexto semântico e semiótico do $\mathrm{KG}$, já que as informações são estruturadas e advindas de diversas fontes e de diversas linguagens, especialmente a híbrida verbal escrita-visual. Outra premissa que pode ser pensada também é se, com o tempo, as pessoas, de maneira geral, irão incorporar o conhecimento KG (hábito) em suas buscas e ele se tornará mais explorado nos resultados de busca, fazendo o leitor permanecer em sua página.

\section{Considerações Finais}

Nos mecanismos de busca, os elementos semióticos e semânticos são inseridos nas SERP a fim de trazer resultados mais relevantes, seja para busca informacional, navegacional ou transacional. Assim, sentido e referência, imagem e palavra, pragmática e semântica reforçam sintaxes computacionais e gramáticas de busca.

Nesse contexto, os sujeitos navegam, pesquisam e buscam suas informações cotidianamente para diversas atividades e em diferentes plataformas, sobrecarregando-se de conteúdo e sofrendo também uma sobrecarga cognitiva. Dessa forma, o estudo de interfaces ou de websites pode tornar-se significativo para avaliar a busca em sistemas de informação.

Em consonância com a orientação desta pesquisa, Sangirardi (2014, p. 228) considera os mecanismos de busca, em especial o Google, como tecnologias cognitivas, dado o "[...] poder de rastreabilidade das atividades cognitivas de seus usuários [...] e seus resultados podem ser considerados como trilhas sígnicas humanas."

Nossa premissa, a partir da Semiótica, foi analisar a influência dos diferentes tipos de signos na escolha dos resultados a partir do tipo de pesquisa, de acordo com Broder (2002), e a partir do strong eye-mind, isto é, a relação entre a visualização de um objeto e o processo cognitivo, utilizando a tecnologia de eye tracking para a realização dos testes com sujeitos. 
Percebeu-se que a influência da imagem pode estimular o comportamento de busca em resultados enriquecidos visualmente (rich snippet). A imagem (hipoícone de primeiridade, mas com proeminência indicial) apresenta proximidade com o mundo real e atrai o olhar dos sujeitos pesquisados, especialmente no rich snippet, mas também no KG, lembrando Santaella $\left(2004\right.$, p. x) ${ }^{6}$ que afirma que as imagens são “[...] pedaços roubados e duplicados da realidade.”.

É certo que o tipo de busca e o hábito influenciam o comportamento de busca e que há um contexto híbrido nesses resultados com a presença do símbolo (linguagem verbal-escrita), mas os resultados com as imagens são os mais escolhidos, de acordo com os estudos de Rodas, Vidotti e Monteiro (2016a). Nesse aspecto, percebeu-se que, na busca informacional - uma data, nos testes de 2015 -, o leitor não precisou navegar e nem linkar para obtê-la. No caso da busca navegacional, houve mais intenções para navegação fora do Google; no entanto, o objetivo do Google, com o KG, é segurar o leitor em sua SERP, fornecendo resultados enciclopédicos, personalizados e contextualizados. Como o próprio blog oficial afirma, ele proporciona encontrar as coisas certas; conseguir os melhores resumos; ir de forma mais ampla e profunda na busca (GOOGLE, 2012).

É certo também que a premissa strong eye-mind, formulada em 1980, apresenta limitações e dificuldades, uma delas, segundo Barreto (2012), é que não é possível pressupor processos cognitivos específicos diretamente a partir de uma visualização particular, ou mesmo a atenção dada pelo sujeito a coisas para as quais não esteja visualizando diretamente, ou seja, a covert attention. No entanto, esse conceito base, associado às teorias cognitivas e padrões estatísticos, pode revelar predileções, hábitos, comportamentos e outros elementos que, se bem analisados, ajudam nos estudos da área, especialmente no ambiente web, confirmando o eye tracking como importante método de pesquisa para a Ciência da Informação.

O comportamento de busca e as tecnologias amigáveis têm sido o objetivo de compreensão e objeto de desenvolvimento, respectivamente, dos engenheiros da Recuperação da Informação, no sentido de apresentar resultados 
"orgânicos", isto é, signos verbais e visuais apresentados de forma natural e cada vez mais híbridos em uma SERP. Nesse sentido, para a Ciência da Informação, esses estudos também podem subsidiar a avaliação e aperfeiçoamento de interfaces, conteúdos e sistemas de informação digitais.

\section{Financiamento}

Bolsa Produtividade Pesquisa (CNPq) - PQ2.

\section{Referências}

ARRAZOLA, Víctor; MARCOS, Mari-Carmen. Fotografía de prensa y redes sociales: la técnica de Eye Tracking. Ambitos: Revista Internacional de Comunicación, Sevilha, n. 27, p. 1-21, 2014.

BARRETO, Ana. Eye tracking como método de investigação aplicado às Ciências da Comunicação. Revista Comunicando, [S.l.], v. 1, n. 1, p. 168-186, 2012.

BATELLE, John. A busca: como o Google e seus competidores reinventaram os negócios e estão transformando nossas vidas. Rio de Janeiro: Elsevier, 2006.

BRODER, Andrei. The Taxonomy of web search. Sigir Forum, [S.l.], v. 36, n. 2, p. 3-10, 2002.

CRUZ, Carla; RIBEIRO, Uirá. Metodologia científica: teoria e prática. 2.ed. Rio de Janeiro: Axcel Books, 2004.

DUCHOWSKI, Andrew. Eye Tracking methodology. 2 ed. Clemson: Springer, 2007.

GOOGLE. The Knowledge Graph. [S.l.], 2012.

JUST, Marcel; CARPENTER, Patrícia. Eye Fixations and Cognitive Processes. Cognitive Psychology, [S.l.], v. 8, p. 441-480, 1976.

HOLMQVIST, Kenneth et al. Eye tracking: a comprehensive guide to methods and measures. Oxônia: Oxford University Press, 2011.

MARCEL, Frank. Rich Snippets - mais informação na Snippet do Google. [S.l.] 13 maio. 2009.

MARCOS, Mari-Carmen; GONZÁLEZ-CARO, Cristina. Comportamiento de los usuarios en la página de resultados de los buscadores: un estudio basado en Eye Tracking. EI Profesional de la Información, [S.l.], v. 19, n. 4, p. 348-358, 2010. 
MAYNES, Rebecca; EVERDELL, Ian. L'évolution des pages de résultats de recherche Google et leurs effets sur le comportement des utilisateurs. [S.l.]: Mediative, 2014.

MONTEIRO, Silvana et al. Em busca da compreensão da "busca" no ciberespaço. In: ENCONTRO NACIONAL DE PESQUISA EM CIÊNCIA DA INFORMAÇÃO, 12., 2011, Brasília. Anais [...]. Brasília: UnB,. p. 2536-2551, 2011.

MONTEIRO, Silvana Drumond. Knowledge Graph e a significação: novos agenciamentos semióticos dos índices contemporâneos. Tendências da Pesquisa Brasileira em Ciência da Informação, João Pessoa, v. 8, n. 2, p. 203-218, 2015.

MONTEIRO, Silvana Drumond; MOURA, Maria. Knowledge Graph e Peirce: uma abordagem semiótica dos índices contemporâneos no ciberespaço. In: TOMAÉL, Maria; ALCARÁ, Adriana (org.). Fontes de informação digital. Londrina: EDUEL. p. 119-146, 2016.

MONTEIRO, Silvana Drumond; MOURA, Maria Aparecida. Knowledge Graph and 'Sematization' in cyberspace: a study of contemporary indexes. Knowledge Organization, [S.l.], v. 41, n. 1, p. 429-439, 2014.

NÖTH, Winfried. Máquinas semióticas. Galáxia, São Paulo, n. 1, p. 51-73, 2001.

ORTIZ-CHAVES, Laura et al. AdWords, imágenes y ceguera a los banners. Un estudio con eye tracking. El profesional de la información, [S.l.], v. 23, n. 3,79-287, 2014.

PEIRCE, Charles Sanders. Collected papers. Cambrigde: Harvard University Press, $c 1931$.

PEIRCE, Charles Sanders. Collected papers. Cambrigde: Harvard University Press, $c 1958$.

PEIRCE, Charles Sanders. Collected papers. Cambrigde: Harvard University Press, $c 1902$.

PEIRCE, Charles Sanders. Semiótica. 4. ed. São Paulo: Perspectiva, 2010.

POOLE, Alex; BALL, Linden. Eye Tracking in human-computer interaction and usability research: current status and future prospects. In: GHAOUI, Claude (ed.). Encyclopedia of Human Computer Interaction. Hershey: Idea Group Reference, p. 211-219, 2006.

RODAS, Cecilio; MARCOS, Mari; VIDOTTI, Silvana. Tecnologia de eye tracking em User Experience. In: ENCONTRO NACIONAL DE GESTÃO, 
POLÍTICAS E TECNOLOGIAS DE INFORMAÇÃO, [1], 2014, Goiânia. Anais: tecnologias da informação aplicadas [...]. Goiânia: UFG, p. 1-8, 2014.

RODAS, Cecílio; VIDOTTI, Silvana. Eye tracking em interface do Google: a influência do elemento "rich snippet". In: SEMINÁRIO EM CIÊNCIA DA INFORMAÇÃO, 6., 2016, Londrina. Anais Eletrônicos [...]. Londrina: UEL, 2016. p.

RODAS, Cecilio; VIDOTTI, Silvana. Eye Tracking em User Experience: o que os seus olhos revelam. Brazilian Journal of information science: research trends, Marília, v.10, n. 3, p.112-119, 2015.

RODAS, Cecílio; VIDOTTI, Silvana; MONTEIRO, Silvana. Eye tracking em interface do Google: novos olhares sobre a influência do "rich snippet" na decisão dos usuários. In: ENCONTRO INTERNACIONAL DE DADOS, INFORMAÇÃO E TECNOLOGIA, 3., 2016, Marília. Anais [...]. Marília: Unesp, 2016a.

RODAS, Cecílio; VIDOTTI, Silvana; MONTEIRO, Silvana. Interface do Google e do Yahoo: a experiência do usuário sob o olhar do eye

tracking. Informação \& Sociedade: Estudos, João Pessoa, v. 26, n. 2, p. 37-50, $2016 b$.

RODAS, Cecílio et al. Encontrabilidade da Informação: uma análise a partir da tecnologia de Eye Tracking. In: ENCONTRO NACIONAL DE PESQUISA EM CIÊNCIA DA INFORMAÇÃO, 17., 2016, Salvador. Anais [...]. Salvador: UFBA, 2016. p. 3791-3810.

SANGIRARDI, Pedro. Tecnologias cognitivas, comunicação e a crise de representação política. Revista Compolítica, Rio de Janeiro, v. 1, n. 4, p. 218234, 2014.

SANTAELLA, Lúcia. Matrizes da linguagem e pensamento: sonora visual verbal: aplicações na hipermídia. 3. ed. São Paulo: Iluminuras: FAPESP, 2005.

SANTAELLA, Lúcia. O que é Semiótica. São Paulo: Brasiliense, 1983.

SANTAELLA, Lúcia. A teoria geral dos signos: como as linguagens significam as coisas. São Paulo: Pioneira, 2004.

SANTAELLA, Lúcia; NÖTH, Winfried. Imagem: cognição, semiótica, mídia. São Paulo: Iluminuras, 2009.

SANTARÉM SEGUNDO, José; SOUZA, Jessica; CONEGLIAN, Caio. Web Semântica: introdução a recursos de visualização de dados em formato gráfico. In: ENCONTRO NACIONAL DE PESQUISA EM CIÊNCIA DA INFORMAÇÃO, 16., 2015, João Pessoa. Anais [...] João Pessoa: UFPB, 2015. p.1-17. 
SAVAN, David. An introduction to C.S. Peirce's full system of semiotic. Toronto: Victoria College of the University of Toronto, 1976.

TOBII. User's Manual Tobii Studio: Version 3.3.1. [S.l.], 2015.

VIDOTTI, Silvana et al. Arquitetura da Informação e Eye Tracking: o que o olhar e os dados revelam. In: ENCONTRO NACIONAL DE PESQUISA EM CIÊNCIA DA INFORMAÇÃO, 17., 2016, Salvador. Anais [...]. Salvador: UFBA, 2016. p. 3736-3754.

WIKIDATA. Sobre a Wikipédia. [S.l.], 2015.

WITTER, Geraldina; PASCHOAL, Giovana. Produção científica na área educacional: realização acadêmica na adolescência. Psicologia em Pesquisa, Juiz de Fora, v. 4, n. 2, p. 135-143, 2010.

\title{
The search and the eye tracking: a semiotic overlook about the knowledge graph
}

\begin{abstract}
The eye tracking is, at the same time, a technology and a research method used in several areas of knowledge and, recently, in the area of Information Science. Its main thesis, formulated by Just and Carpenter (1976), is the strong eye-mind hypothesis, that is a strong relation between visualization and information processing. In this direction, a basic research was done with an experimental design, aiming to evaluate the search behavior in a Search Engine Results Page (SERP) of Google, especially the visualization of the Knowledge Graph (KG) or Knowledge Map. The research proposition was based on the Peirce's Semiotics, in which signs in a search result (SERP), to a lesser or greater extent, can influence choices, qualify results, capture the look and be important or determinant in a choice from a search, explaining the strong eyemind. The results demonstrated that the image is a cognitive attractor; however, the choice also depends on other factors, such as the type of search and the habit of the subject in searches in search engines.
\end{abstract}

Keywords: Eye Tracking. Peirce's Semiotics. Knowledge Graph. Google. Taxonomy of Web Search.

Recebido: $12 / 12 / 2018$

Aceito: $12 / 04 / 2019$

\footnotetext{
${ }^{1}$ Para Peirce, a mente não se trata de uma entidade exclusivamente biológica, mas lógica (PEIRCE, 1931, 1958; SANTAELLA, 2005; NÖTH, 2001; MONTEIRO; MOURA, 2016).

${ }^{2} \mathrm{O}$ Wikidata faz parte do Wikimedia Fountation, uma organização sem fins lucrativos com conteúdo livre. De acordo com o site, o "Wikidata é um banco de dados vinculados [...]"
} 
(WIKIDATA, 2015, online) ou ainda um banco de conhecimento livre que pode ser lido e editado por seres humanos e máquinas.

${ }^{3}$ A notação CP da citação de Peirce significa Collected Papers, e o indicativo 1.302 identifica o número do parágrafo do volume 1 e assim sucessivamente, uma vez que ele não paginava seus textos.

${ }^{4} \mathrm{O}$ experimento foi realizado mediante autorização da Instituição. Os sujeitos participaram de forma voluntária, o consentimento decorreu via documento conhecido como Termo de Consentimento Livre Esclarecido (TCLE) e autorizando a utilização dos dados coletados para fins acadêmicos.

${ }^{5}$ São textos em microformatos com dados estruturados (Markup formats e RDFa) utilizados pelos serviços de busca, no caso o Google, para "[...] oferecer mais informações sobre uma página diretamente em sua listagem de resultados." (MARCEL, 2009, online). No caso, a expressão "rich" qualifica-o como "rico" a partir de inserção de imagens (estrelas de classificação ou fotos), despertando o nosso interesse de investigação a partir da Semiótica.

${ }^{6}$ A paginação em algarismo romano diz respeito às páginas prefaciais do livro citado. 